\title{
Méthodes de lecture des macrotraces sur les céra- miques : comparaison de protocoles d'acquisition des données
}

\section{Methods of reading macrotraces on ceramics: comparison of data ac- quisition protocols}

\author{
Ferrari Mathilde ${ }^{1}$, Giligny François ${ }^{2}$ \\ ${ }^{1}$ Université Paris 1 Panthéon-Sorbonne, mathilde.ferrari27@gmail.com \\ ${ }^{2}$ Université Paris 1 Panthéon-Sorbonne et UMR Trajectoires, francois.giligny@univ-paris1.fr
}

RÉSUMÉ. L'analyse technologique est un des domaines pour lequel les technologies $3 \mathrm{D}$ ont encore beaucoup à apporter. La lecture et l'analyse des stigmates ou macrotraces laissés par les opérations de façonnage et de traitement des surfaces sur les céramiques a été choisie comme application, car elle est encore souvent menée grâce à des observations visuelles ou radiographiques en 2D. C'est une approche comparée qui est proposée ici, par la mise en œuvre sur les mêmes corpus de protocoles d'acquisition et de modélisation 3D par lasergrammétrie, photogrammétrie ou de la RTI (Reflectance Transformation Imaging). Les résultats permettent de dégager des méthodes plus pertinentes que d'autres pour l'analyse des macrotraces.

ABSTRACT. The technological analysis is one of domains for which the 3D technologies have to bring again a lot. The analysis of stigmas or surface markings left by the operations of shaping and surface processing on ceramic was chosen as case study, because it is still often led thanks to visual or radiographic observations in 2D. It is a compared approach which is proposed here, by the implementation on the same corpuses of protocols of acquisition and 3D modelling by lasergrammetry, photogrammetry or the RTI (Reflectance Transformation Imaging). The results reveal methods that are more relevant to macrotrace analysis than others.

MOTS-CLÉS. 3D, photogrammétrie, lasergrammétrie, Reflectance Transformation Imaging, technologie céramique. KEYWORDS. 3D, photogrammetry, lasergrammetry, Reflectance Transformation Imaging, pottery technology.

\section{Problématique et objectifs}

La reconstitution des chaînes opératoires de fabrication est l'un des objectifs de l'analyse technologique des céramiques. Les premiers travaux en ce sens ont consisté à décrire et à comparer les stigmates de façonnage ou de traitement des surfaces (Balfet, 1953, Stevenson, 1953). L'observation des cassures, des irrégularités de surface, de l'orientation des inclusions permettait d'en déduire de la présence de colombins, d'étirements ou de joints. Dans les travaux proposant une méthode systématique de lecture et de description, le concept de «macrotrace», «surface markings» ou «surface features » s'est peu à peu imposé (Rye 1981, Roux 1994 \& 2016, Livingstone-Smith 2001). Limité initialement aux observations de surface le terme de macrotrace décrit désormais par extension tout type d'observation macroscopique ou mésoscopique, que ce soit à la surface, ou à l'intérieur des parois du récipient. Il s'agit de décrire la topographie de surface, des variations de texture, des variations d'épaisseur des parois, la morphologie des cassures, l'orientation de la matrice argileuse, la porosité et les inclusions en vue tangentielle ou radiale. Se pose désormais la question, avec la généralisation de ce type d'observations, de la création d'une nomenclature normalisée, traduite dans plusieurs langues et utilisable par la communauté des chercheurs la plus large possible, à l'image de ce qui a été réalisé pour les industries lithiques.

Par ailleurs, les méthodes permettant de travailler à une échelle d'observation plus fine que la seule observation visuelle ou à faible grossissement, ont été peu à peu développées. Ce sont des radiographies (Rye 1977, Alexander \& Johnston 1982, Carr 1990, Berg 2008), des observations en 
lames minces (Echallier 1984, Rice 1987, Quinn 2013, Ther 2016), la tomodensitométrie (Jansen et al. 2001, Bouzakis et al. 2011, Kahl et Ramminger 2012, Barreau et al. 2014, Karl et al. 2014) ou la réalisation de modèles $3 \mathrm{D}$ afin d'analyser les surfaces et la symétrie des vases en lasergrammétrie ou encore en photogrammétrie (Mara et Sablatnig 2008, Karasik et Smilanski 2008).

Néanmoins, aucun essai comparatif mettant en œuvre ces différentes méthodes sur les mêmes objets n'avait encore été tenté à ce jour. Il a été donc proposé dans le cadre d'un travail universitaire de tester différentes procédures d'acquisition et de confronter leurs résultats (Ferrari 2016).

Dans un premier temps, nous avons cherché à vérifier si les techniques de numérisations que sont la lasergrammétrie et la photogrammétrie permettaient d'obtenir des modèles $3 \mathrm{D}$ de céramique satisfaisants au regard de la lecture des macrotraces. Dans un second temps cette recherche s'est focalisée sur l'utilité et de l'efficacité des modèles numériques pour une analyse céramologique en vue de reconstituer et d'interpréter celles-ci comme des gestes techniques ayant généré ces traces.

\section{Corpus utilisé}

Cette étude a été réalisée sur un corpus de céramique varié comptant des techniques de fabrication et de traitements de surface différents. Ces objets ont été prélevés dans le tessonnier de l'université Paris 1 Panthéon-Sorbonne et en particulier dans les corpus provenant du Proche-Orient qui en constituent l'essentiel. Ce tessonnier regroupe autant des céramiques produites sans l'usage de la force rotative, que des vases tournés ou des vases produits avec des méthodes mixtes, comme une ébauche réalisée au colombin reprise au tour en façonnage secondaire. Cette diversité de macrotraces nous a permis de comparer aux mieux d'une part les différentes techniques de numérisation mais aussi les différentes méthodes de lecture de macrotraces.

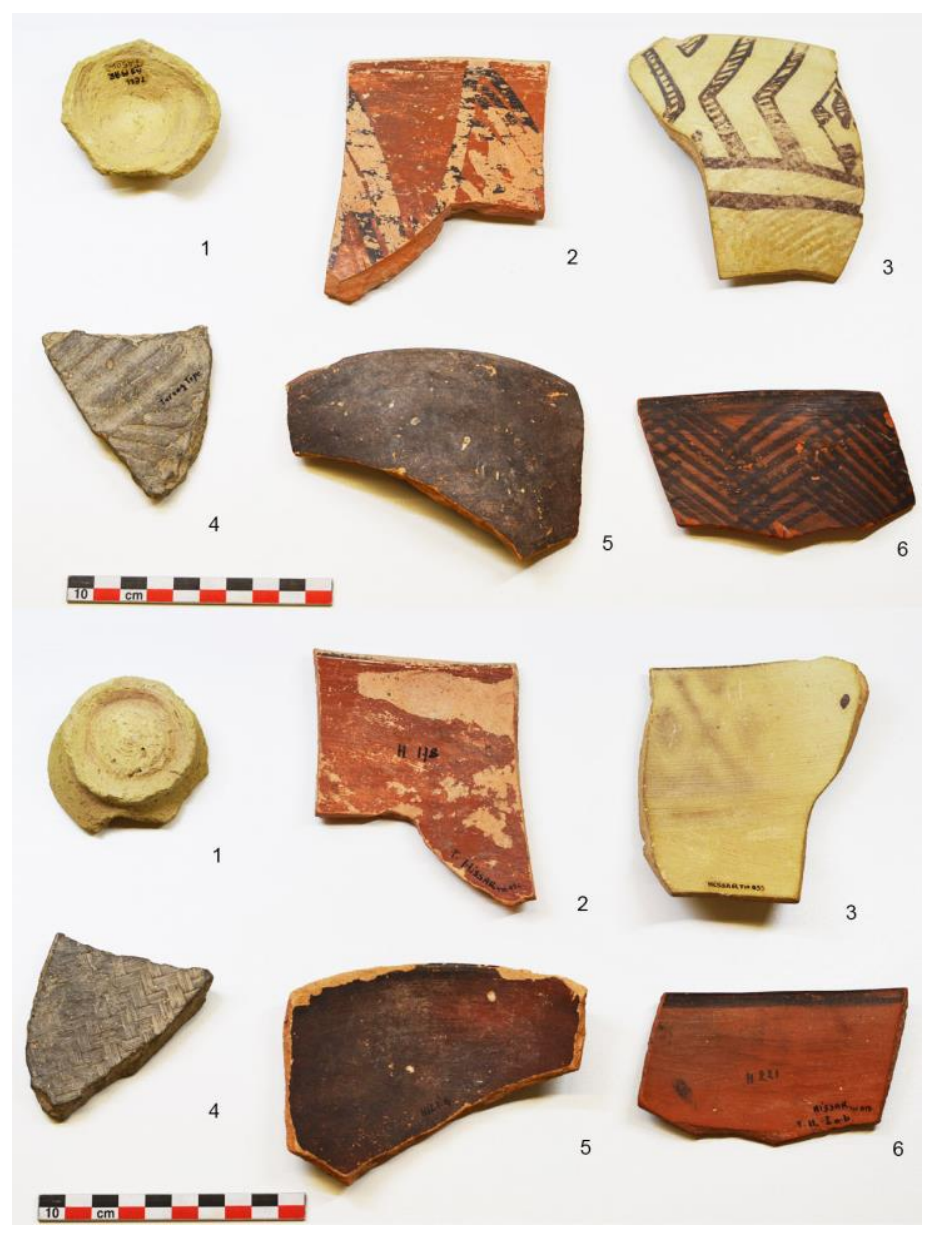

Figure 1. Tessons choisis pour les tests. 1. Tell Asmar TAS002, 2. Tepe Hissar THI94, 3. Tepe Hissar THI55, 4. Tureng Tepe TT128, 5. Hili 6-46. Tepe Hissar THI19. ๑ François Giligny 
Les six échantillons analysés présentent les caractéristiques suivantes (Fig. 1) :

1) Hili6-004: Tesson provenant du site de Hili dans la péninsule d'Oman, aux Emirats arabes unis. C'est une céramique commune avec un décor d'engobe couleur gris-brun, ayant été façonnée par ébauchage aux colombin à joints obliques, ébauche reprise par rotation pour la régularisation et le lissage. Il s'agit d'un bol de $23 \mathrm{~cm}$ de diamètre avec des parois de $3 \mathrm{~mm}$ d'épaisseur. La pâte qui le compose a une matrice argileuse, avec de nombreuses inclusions minérales de fraction sableuse et ne contenant pas de dégraissant végétal. Pour ce qui est des macrotraces, on peut voir les sillons dus à la rotation à l'intérieur du tesson et des traces de pinceau qui a servi à déposer l'engobe. Age du Bronze, céramique sableuse rouge, env. 3000 BC.

2) TAS002 : Tesson provenant du site de Tell Asmar (Irak). Il s'agit d'une base de céramique commune de couleur beige, façonnée grâce à la technique du colombin avec ajout d'un colombin pour le pied. La pâte qui compose ce tesson est une argile carbonatée à texture sableuse comptant des inclusions volcaniques et de quartz en faible quantité. On peut aussi voir l'utilisation de dégraissant végétal. Pour ce qui est des macrotraces présentes sur ce tesson, on peut voir très nettement les différents colombins qui le composent ainsi que la présence de dégraissants végétaux. Age du Bronze env. 3000 BC.

3) TT128 : Tesson provenant du site de Tureng Tepe (Iran). Il s'agit d'une céramique fabriquée par la technique du modelage et étirement sur vannerie. La pâte qui la compose est de couleur grisbleu clair, à la texture fine comprenant quelques inclusions de mica. Les deux faces du tesson présentent des traces : la face externe présente une impression de vannerie liée au montage et la face interne des traces de lissage au lissoir à extrémité mousse assez large (6 à $7 \mathrm{~mm}$ ). Céramique grise de l'âge du Bronze env. 3000 BC

4) THI55 : Tesson provenant du site de Tepe Hissar (Iran). Cette céramique a été fabriquée aux colombins. L'épaisseur est variable sur la hauteur et des ondulations sont perceptibles sur la surface interne. Elle présente une pâte à texture argileuse beige clair avec peu d'inclusions. La lèvre, la partie externe et interne de ce tesson présente un décor peint noir à motifs géométriques, partiellement effacé sur le bord interne. Sur les faces interne et externe on note la présence de micros stries dues au lissage, un partie de la surface externe a été partiellement polie. Des traces d'entailles régulières sont également visibles sur la partie inférieure de la panse peut-être liées à un grattage partiellement effacé par polissage. Painted Buff Ware, Chalcolithique, env. 4000-3500 BC.

5) THI94 : Tesson provenant du site de Tepe Hissar (Iran). Il s'agit d'un bol façonné aux colombins, se composant d'une matrice sableuse rougeâtre comptant quelques inclusions. Intégralement recouvert d'un engobe rouge, sa partie externe présente un décor peint noir à motif géométrique. Les macrotraces présentes sur ce tesson sont des stries dues à une action de raclage et des traces du pinceau ayant servi à appliquer l'engobe ainsi qu'une variation d'épaisseur ou ondulation de la surface, due à la présence de colombins. Painted Red Ware, Tepe Hissar I, Chalcolithique, env. 4300-3700 BC.

6) THI19 : Tesson provenant provient du site de Tepe Hissar en Iran. Il a été façonné à partir de colombins. Sa surface externe présente des traces de lissage ainsi qu'un décor peint aux motifs géométriques. La pâte qui le compte est à texture argileuse rougeâtre sans inclusion ni dégraissant végétal. Les macrotraces visibles en section sont des colombins et en surface des stries dues au lissage. Painted Red Ware, Tepe Hissar I, Chalcolithique, env. 4300-3700 BC. 


\section{Méthodes utilisées}

\subsection{Photogrammétrie}

\subsubsection{Protocoles d'acquisition}

Plusieurs paramètres peuvent influer sur le résultat d'une modélisation: l'appareil photo, l'éclairage, le nombre de clichés, le recouvrement des photographies etc. C'est pourquoi, afin d'optimiser les modèles 3D, plusieurs protocoles ont été mis en place afin de voir en quoi le changement de ces paramètres peut se répercuter sur les résultats de la modélisation (tab. 1).

\begin{tabular}{|c|c|c|c|c|}
\hline & Protocole 1 & Protocole 2 & Protocole 3 & Protocole 4 \\
\hline $\begin{array}{l}\text { Appareil } \\
\text { photographique }\end{array}$ & $\begin{array}{l}\text { Nikon D5200 - } \\
\text { Objectif Focal Fixe } \\
35 \mathrm{~mm}\end{array}$ & $\begin{array}{l}\text { Nikon D750 - } \\
\text { Objectif } \\
\text { 50mm }\end{array}$ & $\begin{array}{l}\text { Nikon D750 - } \\
\text { Objectif Focal Fixe } \\
35 \mathrm{~mm}\end{array}$ & $\begin{array}{l}\text { Nikon D5200 - } \\
\text { Objectif Focal Fixe } \\
35 \mathrm{~mm}\end{array}$ \\
\hline Eclairage & $\begin{array}{l}3 \text { lampes LED à } \\
\text { éclairage neutre }\end{array}$ & $\begin{array}{l}2 \text { panneaux LED } \\
30 \times 30 \mathrm{~cm} 5400 \mathrm{k}\end{array}$ & $\begin{array}{l}2 \text { lampes LED à } \\
\text { éclairage neutre }\end{array}$ & $\begin{array}{l}4 \text { lampes LED à } \\
\text { éclairage neutre }\end{array}$ \\
\hline Mode d'acquisition & Object fixe & Objet fixe & Appareil photo fixe & Appareil photo fixe \\
\hline Logiciel utilisé & Agisoft-Photoscan & Agisoft-Photoscan & Agisoft-Photoscan & Agisoft-Photoscan \\
\hline
\end{tabular}

Tab. 1. Protocoles d'acquisition photogrammétrique mis en œuvre.

\subsubsection{Traitement et essais de modélisation}

Afin de pouvoir utiliser le résultat de ces modèles, il semblait important de pouvoir tout d'abord les remettre à l'échelle. Des grilles de $1 \mathrm{~cm}$ sur $1 \mathrm{~cm}$ ont dont été placées sous chaque céramique afin de pouvoir redimensionner chaque modèle 3D.

Cette opération a été faite sous les logiciels Agisoft-Photoscan et MeshLab. Les modèles générés sous Agisoft-Photoscan peuvent être directement mis à l'échelle par l'utilisation de points de repères sur des axes $\mathrm{x}, \mathrm{y}$ apposés sur la grille de mesure. Cette opération garantit une mise à l'échelle automatique pour laquelle une marque d'erreur est précisée. En revanche, cette mise à l'échelle est opérée manuellement sur le logiciel MeshLab. Ce dernier compte un outil « scale » permettant de changer l'échelle d'un objet sur chacun de ses axes. L'utilisation de cet outil est simple : il suffit de mesurer sur le modèle une distance connue sur notre objet mis à l'échelle. Ici la véritable distance entre ces points est $0.0245 \mathrm{~m}$, cependant, cette distance sur notre modèle non mis à l'échelle est de $23.23 \mathrm{~m}$. Il suffit alors d'effectuer l'opération suivante afin de trouver le coefficient multiplicateur : véritable distance / distance affichée sur le modèle x $100=0.104$. C'est ce coefficient multiplicateur qui sera renseigné pour la mise à l'échelle de chacun des axes.

Le traitement des données des différents tessons numérisés donne les résultats suivants sur trois exemples :

\section{1) Tell Asmar (TAS002) :}

Le modèle du tesson de Tell Asmar présente un niveau de détail satisfaisant, puisque toutes les macrotraces sont présentes sur le modèle 3D et sont très lisibles. Il est alors possible d'utiliser ce modèle pour en faire l'étude céramologique, ce qui montre ici la pertinence de l'utilisation de la photogrammétrie. 


\begin{tabular}{|l|l|l|}
\hline paramètres & Face 1 & Face 2 \\
\hline Nombre de clichés & 30 & 30 \\
\hline Marge d'erreur pour la mise à l'échelle & $0.02 \mathrm{~mm}$ & $0.001 \mathrm{~mm}$ \\
\hline Repères pour l'alignement & 10 & \\
\hline Macrotraces présentes sur la céramique & colombins, vacuoles \\
\hline Macrotraces détectables sur le modèle 3D & colombins, vacuoles \\
\hline Niveau de détail des macrotraces & bon & \\
\hline
\end{tabular}

Tab. 2. Synthèse des macrotraces sur le tesson TAS002 (tab. 2).

\section{2) Tepe Hissar (THI55) :}

Si toutes les macrotraces sont perceptibles sur le modèle 3D de THI55, on observe néanmoins des anomalies dans leur représentation. Les stries sont brouillées par un effet de «peau d'orange » présent sur le modèle et le décor peint se trouve être en relief sur le maillage.

Il ne semble pas pertinent de l'utiliser pour une analyse céramologique au vu du manque de visibilité des macrotraces (tab. 3).

\begin{tabular}{|l|l|l|}
\hline paramètres & Face 1 & Face 2 \\
\hline Nombre de clichés & 42 & 40 \\
\hline Marge d'erreur pour la mise à l'échelle & $0.01 \mathrm{~mm}$ & $0.03 \mathrm{~mm}$ \\
\hline Repères pour l'alignement & 12 \\
\hline Macrotraces présentes sur la céramique & Stries légères dues au lissage, décor peint \\
\hline Macrotraces détectables sur le modèle 3D & Stries légères dues au lissage, décor peint \\
\hline Niveau de détail des macrotraces & Mauvais \\
\hline
\end{tabular}

Tab. 3. Synthèse des macrotraces sur le tesson THI55.

3) Tureng Tepe (TT128) :

Pour ce modèle on observe un décalage entre les deux faces dû à un mauvais alignement manuel.

Le niveau de précision du modèle, hormis ce décalage, est très satisfaisant puisque les décors présents sur chaque côté du tesson sont parfaitement lisibles et permettent d'utiliser ce modèle pour une analyse céramologique (tab. 4).

\begin{tabular}{|l|l|l|}
\hline paramètres & Face 1 & Face 2 \\
\hline Nombre de clichés & 42 & 43 \\
\hline Marge d'erreur pour la mise à l'échelle & $0.03 \mathrm{~mm}$ & $0.5 \mathrm{~mm}$ \\
\hline Repères pour l'alignement & 11 & \\
\hline Macrotraces présentes sur la céramique & Impression de vannerie et traces de lissoir \\
\hline Macrotraces détectables sur le modèle 3D & Impression de vannerie et traces de lissoir \\
\hline Niveau de détail des macrotraces & Très bon \\
\hline
\end{tabular}

Tab. 4. Synthèse des macrotraces sur le tesson TT128.

\subsubsection{Traitement des données}

Mise à l'échelle et comparaison des nuages de points

La comparaison des nuages de points peut être réalisée une fois que nos deux modèles ont été mis à l'échelle. La procédure suivie dans CloudCompare consiste à les ouvrir en format .PSX et à les aligner. L'opération d'alignement se fait manuellement par l'application de point de calages sur les 
deux modèles. Il est alors possible de comparer les écarts existant entre ces deux nuages de points alignés.

L'analyse des nuages de points de ces trois tessons a donné des résultats similaires. On observe donc un écart minime entre ces deux techniques de mise à l'échelle.

Cependant il semble plus pertinent d'utiliser l'outil de mise à l'échelle proposé par MeshLab puisqu'il présente une utilisation beaucoup moins contraignante, en termes de logistique et de manipulation, pour un résultat similaire. En effet, la mise à l'échelle effectuée avec le logiciel Agisoft-Photoscan nécessite la présence lors de la prise de vue d'un carroyage afin de créer les axes $\mathrm{x}, \mathrm{y}$. De plus, le placement des repères sur ces axes peut être une étape longue. Enfin, comme cela a été vu avec la numérisation du tesson de Tureng Tepe cette mise à échelle indépendante des deux faces d'un même objet peu engendre des décalages lors de l'alignement de ces dernières. Il paraît donc plus pertinent en termes de résultat, d'utiliser la mise à l'échelle proposée par le logiciel MeshLab.

\subsubsection{Apports de la photogrammétrie à l'étude céramologique}

Nous avons pu voir grâce au protocole 4 qu'il est possible d'obtenir des modèles 3D très précis, comme pour le tesson THI55, qui présente de légères stries visibles sur son modèle 3D. Cependant, on observe sur l'ensemble de nos modèles un effet d'aspérité qui peut venir brouiller certaines macrotraces et rendre difficile l'étude céramique.

Après avoir modélisé des tessons, dont la précision facilite la reconnaissance des macrotraces et ainsi l'étude les techniques de façonnage et de traitement de surface, il semble maintenant important de discuter de l'apport effectif de la 3D à l'étude céramologique. Dans un premier temps, l'une des utilisations possible de la modélisation 3D est à des fins de simplification du dessin scientifique. Grâce au logiciel CloudCompare, il a pu être possible d'effectuer plusieurs sections grâce à l'outil Extract cloud section. Une fois ces coupes créées il est alors possible d'en faire un dessin vectoriel sur un logiciel de dessin vectoriel comme Adobe Illustrator ® (Fig. 2). Cette utilisation permet d'autre part de pouvoir contrôler la véracité de ce dessin. Puisque la chaîne opératoire de la création de ce dessin est uniquement réalisée par CloudCompare, il n'y a pas d'erreur possible dans ce dernier dès lors que la véracité de notre modèle 3D est vérifiée.
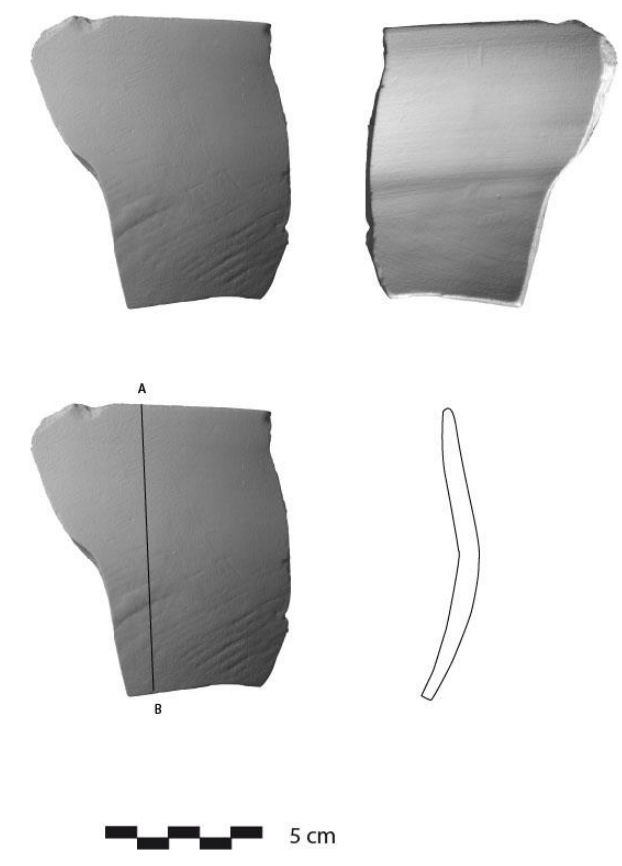

Figure 2. Coupe du tesson Tell Asmar générée grâce au logiciel CloudCompare. CMathilde Ferrari. 
D'autre part, il est possible d'effectuer des orthophotographies à l'aide du logiciel AgisoftPhotoscan. Cependant ces dernières ne peuvent être calculées qu'en fonction de vues manuelles définies, ce qui pose un problème quant à la véracité et la pertinence de ces ortho-images. La combinaison de ces données permet la réalisation de planches entièrement réalisées à partir de la 3D (Fig. 2).

\subsection{Lasergrammétrie}

\subsubsection{Matériel utilisé et mode d'acquisition}

Pour cette procédure, les céramiques ont été analysées à l'aide du scanner laser 3D Minolta Range 7. L'utilisation de ce scanner a ainsi permis de générer des modèles 3D qui ont pu être comparés aux modèles obtenus en photogrammétrie.

Ce scanner est composé de 5 lasers et d'une caméra. Il suit le même principe de fonctionnement utilisé par le scanner laser 3D NextEngine présenté précédemment. En effet, ici le Minolta Range 7 projette 5 rayons laser sur l'objet étudié, qui vont ainsi balayer sa surface. Les déformations des rayons sont alors perçues et analysées par une caméra intégrée au laser, qui permettra ainsi de restituer la forme de l'objet. Le processus de numérisation n'étant pas automatisé à l'aide d'un plateau rotatif synchronisé au scanner, il est nécessaire d'effectuer manuellement plusieurs scannérisations d'un même objet sous différents angles. Cependant, cette opération est simplifiée par l'utilisation de cibles reconnues par le scanner et permettant ainsi d'aligner automatiquement nos scans. Pour cela, il est nécessaire d'apposer à côté et/ou sur notre objet des cibles ne devant pas bouger pendant toute la durée de l'acquisition. L'acquisition se fait à l'aide du logiciel RangeViewer dans lequel il est nécessaire d'informer le mode de prise de vue, ici à l'aide de cibles. Une fois les paramètres de précision définis il est possible de démarrer l'acquisition. Pour cela, il est possible de générer un aperçu de notre scan afin de vérifier d'une part si notre objet est bien positionné, permettant ainsi sa numérisation et, d'autre part, de vérifier que le scanner reconnaît les cibles apposées. Il est important que le scanner les reconnaisse afin de permettre l'alignement automatique des scans. Si l'aperçu généré semble satisfaisant, il est nécessaire d'effectuer le scan. Une fois ce scan fini, l'objet doit être tourné en le positionnant de façon à ce que les cibles déjà reconnues apparaissent dans ce nouveau scan et ainsi permettre son alignement. Toute l'acquisition suit donc un cheminement lié à la position des cibles.

\subsubsection{Numérisation et résultats}

1) THI55 : Ce premier tesson, numérisé en deux fois, a livré des résultats très intéressants au regard des macrotraces. En effet on peut observer sur ce modèle que les légères stries présentes sur les faces internes et externes du tesson sont très bien figurées sur le modèle $3 \mathrm{D}$.

De plus, au vu de la précision du scanner, on constate très peu d'effet « peau d'orange » présent sur les modèles générés en photogrammétrie et venant brouiller les macrotraces visibles. Cependant, comme cela a été observé sur les modèles générés à l'aide du scanner NextEngine, ainsi qu'en photogrammétrie, le décor peint présent sur le tesson est interprété ici aussi comme étant une variation de surface et est très nettement visible sur le maillage (Fig. 3). De plus, si les cibles permettent un alignement des scans on peut remarquer ici que cet alignement devient moins précis dès lors que la surface de l'objet s'éloigne de ces cibles. En effet, si notre maillage ne présente pas d'écart entre les scans à la base de notre tesson, on peut remarquer un écart sur les parties les plus éloignées des cibles. Enfin, le scanner Minolta Range 7 ne générant pas de texture, l'alignement du maillage s'en est retrouvé compliqué par le manque de points similaires entre les deux modèles du tesson. Si les modèles générés grâce à cette technique sont très précis au regard des macrotraces, on peut remarquer des artefacts présents sur le maillage et un écart de l'alignement, qui ne permettent pas l'étude céramologique de ce tesson au moyen de ce modèle 3D 


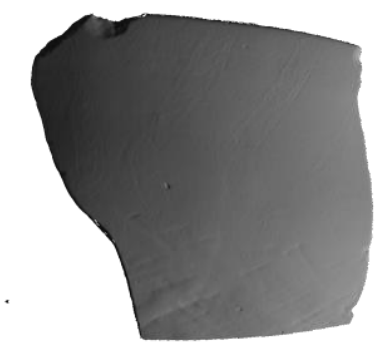

Figure. 3. Présence du décor peint sur le tesson THI55 ๑ Mathilde Ferrari

2) Tureng Tepe (TT128) : Ce second tesson numérisé en deux fois a nécessité plusieurs manipulations. En effet, afin que toutes les cibles soient reconnues par le logiciel de numérisation, il est parfois nécessaire d'effectuer des scans de transition. Ces scans peuvent avoir un angle rasant trop important avec une des faces du tesson. Les points générés sur ces faces, moins précis, vont ainsi parasiter le maillage final (Fig. 3). C'est pourquoi, il a été nécessaire ici de supprimer les numérisations de transition afin d'obtenir un maillage composé uniquement de données précises. Le résultat est finalement très satisfaisant, puisque l'on obtient un maillage très net de notre tesson. De plus, ce tesson n'étant pas grand, la distance entre la base de ce dernier et ses parties les plus hautes ne sont pas importantes. On ne constate pas alors d'écart entre les différents scans, l'ensemble du tesson étant suffisamment proche des cibles de calage. Enfin, même si aucune texture n'est générée par le scanner laser, ici l'alignement des deux modèles a été facilité par la présence du décor présent sur le maillage. Pour ce qui est de ce tesson, cette technique de numérisation s'est relevée être très pertinente.

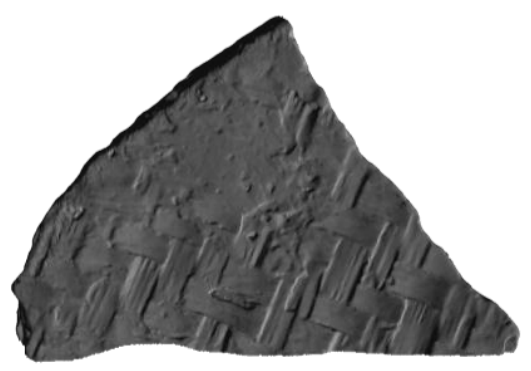

Figure 4. Maillage du tesson de Tureng Tepe TT128, face externe. ๑ Mathilde Ferrari.

3) Tepe Hissar (THI94) : Pour ce dernier tesson numérisé en deux parties, il a aussi été nécessaire comme pour Tureng Tepe d'effectuer des traitements du premier maillage généré. En effet, ici aussi la numérisation a nécessité la présence de scans intermédiaires qui ont généré des points venant parasiter le maillage. De plus, on peut observer sur le maillage une fois nettoyé la présence du décor peint interprété ici comme étant en relief, ainsi qu'un léger écart dans les parties hautes de ce dernier (Fig. 4). Au vu des résultats obtenus pour la numérisation de ce tesson, il n'a pas été jugé nécessaire d'assembler les deux modèles permettant de le reconstituer.

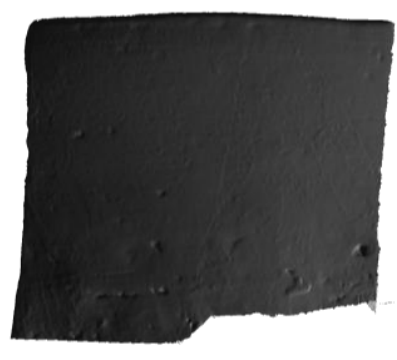

Figure 5. Maillage du tesson de Tepe Hissar (THI94) après traitement. 
Au regard des résultats obtenus par les numérisations effectuées avec le scanner Minolta Range 7 et bien que certaines manipulations restent à faire pour pallier aux erreurs d'alignements des scans, il semble que cette méthode se prête parfaitement à la numérisation des céramiques. Cependant, comme pour tous les scanners utilisés ici, il ne convient pas pour les tessons présentant des variations de couleurs trop contrastées.

\subsubsection{Comparaison des nuages de points}

Dans un premier temps, afin de pouvoir comparer les nuages de points, il a fallu assembler nos deux faces de chaque tesson. Cette opération a été faite grâce au logiciel MeshLab. Il a été nécessaire d'apposer 10 repères afin d'obtenir un alignement des plus précis possible. Cet alignement a posé des difficultés, compte tenu du manque de texture. Fort heureusement, le maillage étant assez précis il a été possible d'apposer ces repères sur certaines inclusions présentes, ainsi que sur le relief du décor peint présent sur le maillage.

Une fois cet alignement effectué, il a fallu toujours sur le logiciel MeshLab, mettre à l'échelle notre maillage.

La comparaison des nuages de points s'est effectuée grâce au logiciel CloudCompare, grâce auquel on a pu observer un écart minime entre le modèle généré en lasergrammétrie et en photogrammétrie (Fig. 3). La zone sur laquelle les écarts sont le plus importants est au niveau du décor peint du tesson THI55, qui est bien plus marqué sur le modèle généré en lasergrammétrie. Il est donc normal d'observer plus de variations dans cette zone. Cependant, aucun écart majeur n'est à noter, compte tenu des techniques de numérisation, d'alignement et de mise à l'échelle différentes.

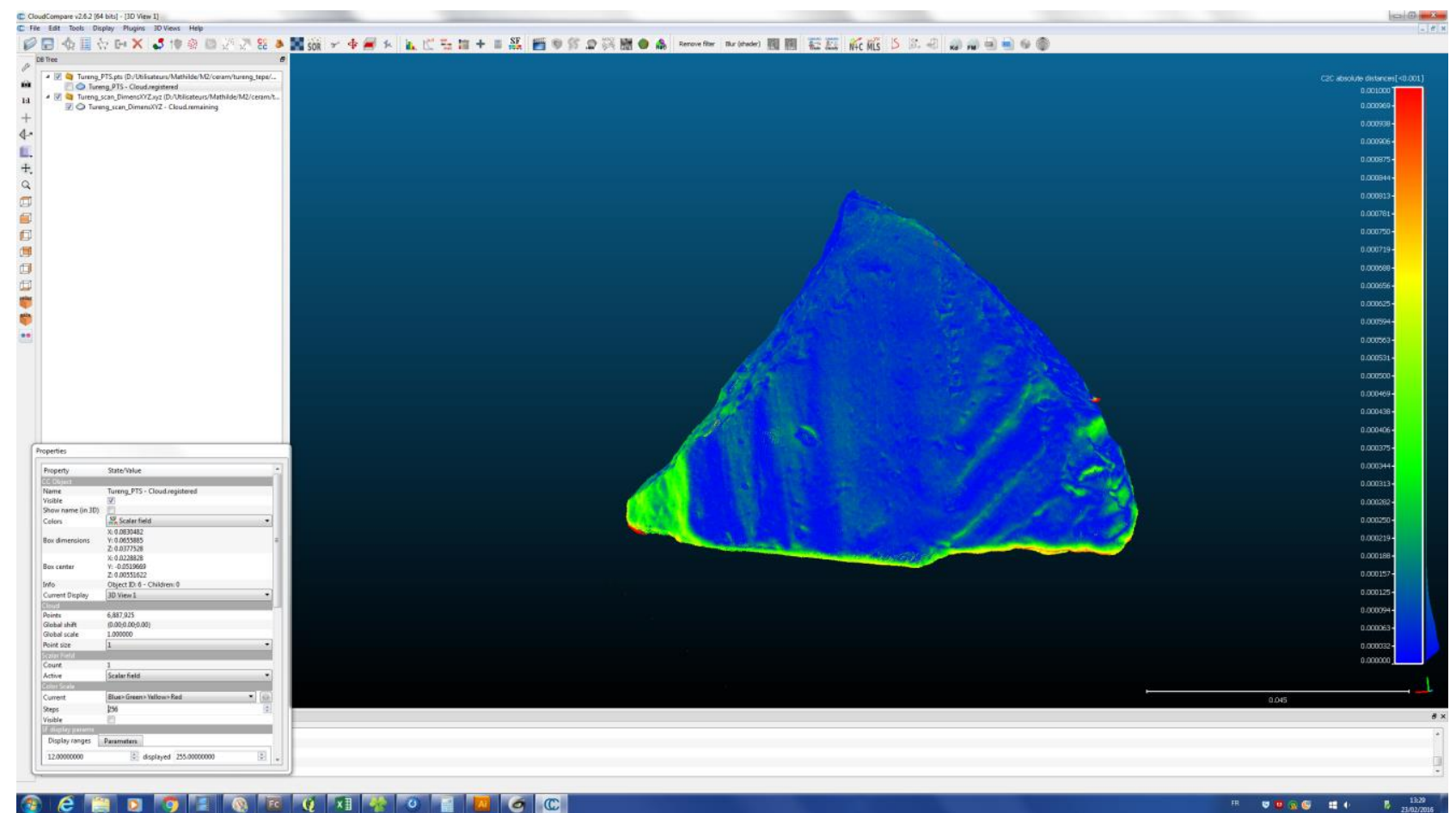

Figure 6. Comparaison des nuages de points générés en lasergrammétrie et photogrammétrie du tesson TT128. OMathilde Ferrari.

\subsection{Reflectance Transformation Imaging (RTI)}

En ce qui concerne l'étude des macrotraces, il semble important de mentionner la technique photographique de la Reflectance Transformation Imaging ou RTI développée par le Cultural Heritage Imaging ${ }^{1}$. Cette technique permet de regrouper plusieurs photos d'un même objet effectuées avec des éclairages différents, afin de générer un document 2D modulable permettant de 
changer l'éclairage de ce même objet (Earl et al. 2011). Le rendu de cette technique permet donc de faire apparaitre un objet sous différents éclairages et ainsi y faire figurer les différents détails présents sur ce même objet.

\subsubsection{Protocole d'acquisition et de traitement}

Cette technique nécessite la mise en place d'un protocole d'acquisition assez lourd. En effet, l'appareil photographique est positionné de manière zénithale au-dessus de l'objet étudié. Deux sphères doivent être placées de part et d'autre de ce dernier, car c'est grâce à ces sphères que le logiciel de traitement peut positionner les différents éclairages. Il est préférable que la taille de ces dernières soit adaptée à la taille de l'objet. Plus celui-ci sera petit, plus les sphères devront l'être. Une fois l'appareil photographique, les sphères, ainsi que l'objet ont été positionnés, l'acquisition peut commencer. Il est primordial que ni l'appareil photo, ni l'objet ne bougent durant cette acquisition. C'est pourquoi il est nécessaire de prendre les photos à l'aide d'un déclencheur à distance.

Enfin, il est nécessaire que la source de lumière soit mouvante. Cet éclairage doit s'effectuer en un balayage régulier, allant d'une lumière rasante à zénithale en plusieurs points sur l'objet.

Cette opération a été réalisée au sein du studio photographique du C2RMF du Louvre avec l'appareil photo Nikon D4S, un objectif à focal fixe Nikon de 35mm ainsi qu'un flash Nikon SB700 .

L'acquisition de la RTI est dans son principe similaire à la photogrammétrie. Ici le flash doit être placé à distance égale pour 10 positions autour de l'objet et sur chacune de ces positions observées à une altitude différente, d'une position zénithale à rasante. Cette technique nécessite donc une mise en place complexe ainsi que l'acquisition de matériel tel qu'un déclencheur à distance, ainsi qu'un flash connecté à l'appareil photographique. Pour chaque objet 50 clichés en moyenne sont nécessaires afin d'obtenir des résultats satisfaisants. Pour générer notre document, les photographies sont exportées vers le logiciel RTI sur lequel très peu de manipulations sont à faire. Seule la position des sphères doit être renseignée, afin de permettre au logiciel de positionner les photos. En effet, c'est grâce au reflet du flash dans ces sphères que le logiciel calcule la position de chaque photographie. Une fois le document calculé, il est ouvert dans le logiciel RTIViewer. Il est alors possible de changer la position de l'éclairage, mais aussi certains paramètres comme la saturation des images permettant ainsi de mettre en évidence certains détails peu visibles sur l'objet.

\subsubsection{Résultats}

Un tesson a fait l'objet d'une acquisition et d'un traitement par RTI particulièrement efficace, le tesson THI55.

Les résultats obtenus sont très satisfaisants puisqu'ils permettent une étude visuelle des macrotraces très détaillée. En effet cette technique permet de mettre en évidence des détails peu perceptibles à l'œil nu comme le montrent les macrotraces présentes sur le tesson THI55 (Fig. 6). En effet, les légères stries présentes sur THI55, sont parfaitement visibles grâce à cette technique et bien plus lisibles que lors d'un examen à l'oeil. Il est aussi important de rappeler que pour ce tesson les macrotraces sont ici bien mieux détaillées que sur les modèles 3D générés en photogrammétrie et lasergrammétrie. De plus, si l'utilisation du logiciel de calcul est simple, la mise en place de la procédure d'acquisition reste elle bien plus complexe que pour ces deux dernières techniques. En effet, l'utilisation de la RTI impose une staticité totale de l'objet étudié et de l'appareil photographique qui nécessite l'acquisition de matériels particuliers parfois couteux et si possible un véritable studio. 


\begin{tabular}{|l|l|l|}
\hline paramètres & Face 1 & Face 2 \\
\hline Nombre de clichés & 45 & 45 \\
\hline Nombres de sphères & 2 & 2 \\
\hline Macrotraces présentes sur le tesson & Stries légères, décor peint & \\
\hline Macrotraces détectables sur le modèle RTI & Stries légères, décor peint \\
\hline Visibilité & Très bonne & \\
\hline
\end{tabular}

Tab. 5. Synthèse des macrotraces sur le tesson THI55.

Enfin, au regard des macrotraces et de leur analyse visuelle simple, la RTI supplante largement l'utilisation de la 3D, comme cela a pu être montré avec l'étude du tesson THI55. Son utilisation permet donc de générer des supports de travail 2D, ainsi que de créer des illustrations très parlantes. Cependant, cette technique ne permet pas d'extraire de données métriques ainsi que des dessins de coupes comme le permet l'utilisation de la 3D.
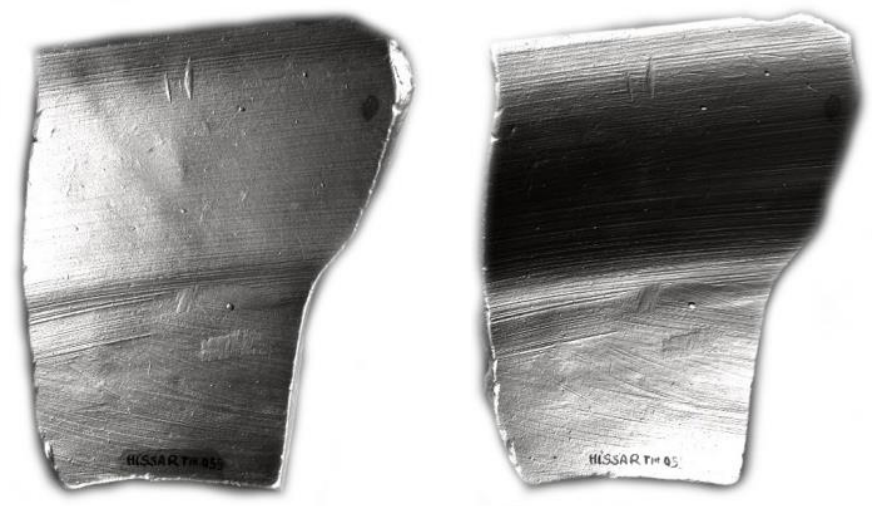

Fig. 7. Macrotraces visibles sur le tesson THI55 en RTI. CMathilde Ferrari.

\section{Conclusion}

A l'issue de ces différents essais, l'enseignement que l'on peut en retirer est qu'il convient d'appliquer la technique de numérisation en fonction des informations que l'on souhaite obtenir.

La photogrammétrie et la lasergrammétrie permettent d'acquérir des nuages de points et de manipuler ces données, de créer des sections, des vues 2D et des orthophotographies, de calculer des distances, des profondeurs, et enfin d'obtenir une empreinte numérique des traces.

La RTI peut mettre en évidence des stigmates visuellement, mais sans pouvoir ensuite permettre de traiter les données par le calcul.

On retrouve ici, pour la lecture des céramiques, certaines problématiques liées à l'art pariétal et à l'analyse des traces sur des supports gravés, sculptés ou peints. La recherche et la confrontation de protocoles d'acquisition ont été également réalisés en vue d'analyses tracéologiques par photomicrographie ou photogrammétrie (Plisson 2015, Plisson et Zodkina 2015). La lasergrammétrie, la photogrammétrie et la photographie oblique ont été mises en œuvre pour l'analyse de gravures dans des tombes mégalithiques (Cassen et Robin 2010, Cassen et al. 2014) ou récemment encore, la RTI (Díaz-Guardamino et al. 2015).

Le développement de ce type de tests devra être poursuivi à l'avenir, afin de pouvoir définir les procédures et les méthodologies les plus adaptées aux besoins des chercheurs, que ce soit en termes 
d'analyse des données par le traitement des nuages de points ou des images, que de rendu sous la forme de modèles 3D ou d'images 2D. La céramologie est à ce titre un champ d'expérimentation encore largement peu exploré. Nous pourrons envisager alors de documenter technologiquement des séries de référence, archéologiques, expérimentales ou ethnographiques à l'aide des techniques de la 3D et de l'imagerie. A terme, il peut être envisagé de créer des atlas en ligne permettant une meilleure comparaison des données et une interprétation des macrotraces sur la base d'une documentation élargie et consultable par tous.

\section{Remerciements}

Ce travail a été en partie réalisé lors d'un stage de Master professionnel d'archéologie à l'université Paris 1 Panthéon-Sorbonne sous la direction de François Giligny et réalisé au sein du Centre de Recherches des Musées de France et nous souhaitons remercier ici tout particulièrement Mr Nicolas Melard et Mme Anne Maigret qui nous ont accueillis et ont suivi notre travail. Nous remercions également Mr Laurent Aubry de l'UMR Trajectoires pour son encadrement et ses conseils.

\section{Notes:}

1. http://culturalheritageimaging.org/Technologies/RTI/

\section{Références :}

ALEXANDER, R. E., \& JOHNSTON, R. H. 1982. Xeroradiography of ancient objects: a new imaging modality. In J. S. Olin and A. D. Franklin (eds.), Archaeological ceramics (). Washington, DC: Smithsonian Institution, p. 145154.

BALFET H. 1953. Notes sur le façonnage des poteries préhistoriques. Bulletin de la Société Préhistorique Française, L, p. 211-217.

BARREAU J.-B., NICOLAS T., BRUNIAUX G., PETIT E., PETIT Q., BERNARD Y., GAUGNE R., GOURANTON V., 2014. Ceramics Fragments Digitization by Photogrammetry, Reconstructions and Applications. International Conference on Culturage Heritage, EuroMed, 2014, Nov 2014, Lemessos, Cyprus.

BERG (I.) 2008. Looking through pots: recent advances in ceramics X-radiography, Journal of Archaeological Science, 35, 2008, p. 1177-1188.

BOUZAKIS D.-K., PANTERMALIS D., EFSTATHIOU K, VARITIS E., PARADISIADIS G. \& MAVROUDIS I., 2011. An Investigation of Ceramic Forming Method Using Reverse Engineering Techniques: The Case of Oinochoai from Dion, Macedonia, Greece, Journal of Archaeological Method \& Theory, 18, p. 111-124.

CARR, C., 1990. Advances in ceramic radiography and analysis: applications and potentials. Journal of Archaeological Science, 17, p. 13-34.

CASSEN S., ROBIN G., 2010. Recording Art on Neolithic Stelae and Passage Tombs from Digital Photographs, Journal of Archaeological Method and Theory, 17, p. 1-14.

CASSEN S., LESCOP L., GRIMAUD V., ROBIN G., 2014. Complementarity of acquisition techniques for the documentation of Neolithic engravings: lasergrammetric and photographic recording in Gavrinis passage tomb (Brittany, France). Journal of Archaeological Science, 45, p. 126-140.

DÍAZ-GUARDAMINO M., SANJUÁN L. G., WHEATLEY D., ZAMORA V. R., 2015. RTI and the study of engraved rock art: A re-examination of the Iberian south-western stelae of Setefilla and Almadén de la Plata 2 (Seville, Spain), Digital Applications in Archaeology and Cultural Heritage, 2(2-3), p. 41-54.

EARL, G., BASFORD, P. J., BISCHOFF, A. S., BOWMAN, A., CROWTHER, C., DAHL, J., HODGSON, M., MARTINEZ, K., ISAKSEN, L., PAGI, H., PIQUETTE, K. E. AND KOTOULA, E., 2011. Reflectance transformation imaging systems for ancient documentary artefacts. In : Bowen J. P., Dunn S. and Kia Ng (eds.) EVA London 2011: Electronic Visualisation and the Arts, BCS. 
FERRARI M. 2016. Applications de la 3D en archéologie : acquisition et traitement. Mémoire de Master Professionnel d'archéologie de l'université Paris 1-Panthéon-Sorbonne.

JANSEN, R., KOENS, H., NEEFT, C., \& STOKER, J. 2001. Scenes from the past: CT in the archaeology of ancient Greek ceramics. RadioGraphics, 21, p. 315-321.

KARL S., JUNGBLUT D., MARA H., WITTUM G. et KRÖMKER S. 2014. Insights into manufacturing techniques of archaeological pottery: Industrial X-ray computed tomography as a tool in the examination of cultural material. In M Martinón-Torres (Ed.), Craft and science: International perspectives on archaeological ceramics. Doha, Qatar, Bloomsbury Qatar Foundation. p. 253-261.

KAHL W.-A., RAMMINGER B. 2012. Non-destructive fabric analysis of prehistoric pottery using high-resolution Xray microtomography: a pilot study on the late Mesolithic to Neolithic site Hamburg-Boberg, Journal of Archaeological Science 39, p. 2206-2219.

KARASIK, A., SMILANSKI, U., 2008. 3D scanning technology as a Standard archaeological tool for pottery analysis: practice and theory. Journal of Archaeological Science, 35, p. 1148-1168.

LIVINGSTONE-SMITH A., 2001. Chaînes opératoires de la poterie. Références ethnographiques, analyse et reconstitution. Doctoral Thesis, Bruxelles: Faculté de Philosophie et Lettres, Université Libre de Bruxelles.

MARA, H. and R. SABLATNIG 2008. Evaluation of 3D Shapes of Ceramics for the Determination of Manufacturing Techniques, in: Posluschny, A., K. Lambers and I. Herzog (eds.), Layers of Perception. Proceedings of the 35th International Conference on Computer Applications and Quantitative Methods in Archaeology (CAA), Berlin, Germany, April 2-6, 2007 (Kolloquien zur Vor- und Frühgeschichte, Vol. 10). Dr. Rudolf Habelt GmbH, Bonn, p. 9197.

PLISSON H., 2015. Digital Photography In Use-Wear Studies, From 2d To 3d, In : Vergnieux R. et Delevoie C., (eds.), Actes du Colloque Virtual Retrospect 2013, Archéovision 6, Editions Ausonius, Bordeaux, p. 35-47.

PLISSON H. et ZODKINA L. V., 2015. From 2D TO 3D at macro and microscopic scale in rock art studies, Digital Applications in Archaeology and Cultural Heritage, 2 (2015), p. 102-119.

QUINN P., 2013. Ceramic Petrography: The Interpretation of Archaeological Pottery \& Related Artefacts in Thin Section, Archaeopress, Oxford.

ROUX, V. 1994. La technique du tournage : définition et reconnaissance par les macrotraces. In (D. Binder \& J. Courtin, Eds) Terre Cuite et Société. La Céramique, Document Technique, Economique, Culturel. Juan-les-Pins: Editions APDCA, p. 45-58.

ROUX V. 2016. Des céramiques et des hommes. Décoder les assemblages archéologiques. Presses universitaires de Paris Ouest.

RYE, O. S. 1977. Pottery manufacturing techniques: X-ray studies. Archaeometry, 19(2), p. 205-211.

RYE O.S. 1981. Pottery technology. Manuals of Archaeology, vol. 4, Taraxacum, Washington.

STEVENSON R. B. K., 1953. Prehistoric Pot-Building in Europe, Man, Vol. 53, May, 1953, p. 65-68.

THER R. 2016. Identification of Pottery-Forming Techniques using Quantitative Analysis of the Orientation of Inclusions and Voids in Thin Sections, Archaeometry 58 (2), p. 222-238. 